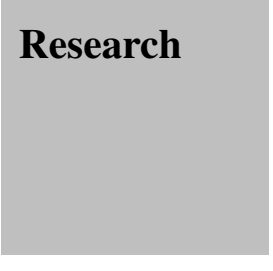

\title{
Reliability Estimation Based on Operational Data of Manufacturing Systems
}

\author{
Lin $\mathrm{Li}^{1, *} \dagger$ and $\mathrm{Jun}^{\mathrm{Ni}^{2}}$ \\ ${ }^{1}$ Department of Mechanical Engineering, University of Michigan-Ann Arbor, 1035 H. H. Dow, 2300 Hayward Street, \\ Ann Arbor, 48109-2136 MI, U.S.A. \\ ${ }^{2}$ Department of Mechanical Engineering, University of Michigan-Ann Arbor, 1023 H. H. Dow, 2300 Hayward Street, \\ Ann Arbor, 48109-2136 MI, U.S.A.
}

\begin{abstract}
Maintenance management has a direct influence on equipment reliability and safety. However, a large portion of traditional maintenance models and reliability analysis methods usually assumes that only perfect maintenance is performed on the system and the system will restore to as good as new regardless of the kind of preventive maintenance work-order that is performed. This is not practical in reality and may result in an inaccurate parametric estimation. The research objective of this paper is to develop a maximum likelihood estimation method to obtain more accurately estimated parameters based on the operational data of manufacturing systems, taking into consideration the difference between perfect and imperfect maintenance work-orders. Weibull distribution is specifically studied for this purpose. A practical case study based on industrial operational data from an automotive assembly line is performed to illustrate the implementation and efficiency of the proposed reliability estimation method. Copyright $\odot 2008$ John Wiley \& Sons, Ltd.
\end{abstract}

KEY WORDS: operational data; reliability estimation; preventive maintenance; maximum likelihood estimation

\section{INTRODUCTION}

7 echnically, reliability has often been defined as the probability of a system, vehicle, machine, device, and so on, to perform its intended function under operating conditions for a specified period of time ${ }^{1}$. Many models and methods have been used for the reliability estimation problem in different fields ${ }^{2,3}$.

The two-parameter Weibull distribution is widely used for modelling of the failure time in life testing and reliability theory ${ }^{4}$. Various methods have been proposed for the estimation of the Weibull parameters: the maximum likelihood estimation (MLE) method is powerful in terms of the theoretical prospective, whereas the least squares estimation (LSE) method is computationally easier to handle and provides simple closed-form estimated solutions ${ }^{4}$. Generally, the bigger the samples, the higher the accuracy of the estimates.

\footnotetext{
${ }^{*}$ Correspondence to: Lin Li, Department of Mechanical Engineering, University of Michigan—Ann Arbor, 1035 H. H. Dow, 2300 Hayward Street, Ann Arbor, 48109-2136 MI, U.S.A.

†E-mail: lilz@umich.edu
} 
Statistical theory ${ }^{1,5,6}$ shows that maximum likelihood estimators are 'optimal' in large samples. Zhang et al. $^{7}$ proposed the robust regression (RR) method that has been verified to work better than the least square regression method for small data sets. The MLE on comparison with the RR does not show strong evidence of better estimation for small data sets. While dealing with complete, small samples, estimators of MLE and LSE have been found to be biased ${ }^{8}$. A great deal of research has been conducted on the topics of bias correction for the maximum likelihood estimator ${ }^{9-11}$ and least squares estimator ${ }^{12}$ of Weibull parameters.

Besides the Weibull distribution, the theories of reliability estimation utilizing the failure-time data and censored data have been systematically constructed ${ }^{1}$. However, these kinds of data are not enough to describe the reliability performance of equipment on the plant floor because maintenance work-orders are widely performed in the plants and have great effect on equipment reliability.

The goal of maintenance is to improve system availability and reliability in order to reduce the downtime and frequency of failures and to minimize the total cost of system operation. Generally, maintenance may be categorized into two major classes: reactive maintenance (RM) and preventive maintenance (PM). RM is performed when a machine fails. This usually involves replacing or repairing of the components that are responsible for the failure of the overall system in order to restore the system functionality. PM involves the replacement or repair of system components before they fail. The objective of PM is to enable continuous uninterrupted system production.

Many existing maintenance models usually assume that only perfect maintenance is performed on the system and the system will restore to as good as new after PM. This is not practical in reality ${ }^{13}$. Based on the restoration type, PM may be classified into minimal maintenance $(\mathrm{mm})$ and major maintenance $(\mathrm{mm})^{14,15}$. These two maintenance categories are explained in literature as follows.

- Minimal maintenance: Maintenance of limited effort and effect. For deterioration modelled as a discrete stage, $\mathrm{mm}$ restores the system to the previous deterioration stage. This is imperfect maintenance.

- Major maintenance: Maintenance wherein a system is restored to good-as-new. This is perfect maintenance.

Therefore, these two maintenance types have different influences on the equipment reliability performance. The present work will incorporate information on maintenance category ( $\mathrm{Mm}$ or $\mathrm{mm}$ ) into reliability estimation based on the MLE method. An industrial case study is performed to illustrate the implementation and the efficiency of the proposed method on the plant floor.

The remainder of this paper is organized as follows. Section 2 introduces the general procedure of dealing with the operational data, including the failure-time data, and maintenance category data: $\mathrm{mm}$ and $\mathrm{Mm}$ records, to perform reliability estimation using the MLE method. Section 3 presents the numerical case study. In Section 4, an industrial case study utilizing the operational data logs from a real production line of an automotive assembly plant is performed. Section 5 discusses the conclusions and directions for future work.

\section{METHOD DESCRIPTION}

\subsection{Data explanation}

Operational data including times of machine stoppage and failures, as well as maintenance actions, are important and information-rich outputs from the production lines. Based on the operational data, equipment reliability parameters can be estimated with higher confidence than when it is estimated only through a limited number of runs of failures. Most current data collection instruments on the plant floor can record the failure-time data and PM work-orders finished. Failure usually results in RM, restoring the equipment to a perfect reliability condition (the reliability value is equal to one). The current reliability estimation method on the plant floor simply treats all PM tasks record as right-censored data, which means that the equipment reliability is restored to one regardless of the kind of PM task that is performed.

However, $\mathrm{mm}$ and $\mathrm{Mm}$ have different effects on equipment reliability performance. Generally, the $\mathrm{Mm}$ can be considered as right-censored data when used for reliability analysis, as the equipment reliability 


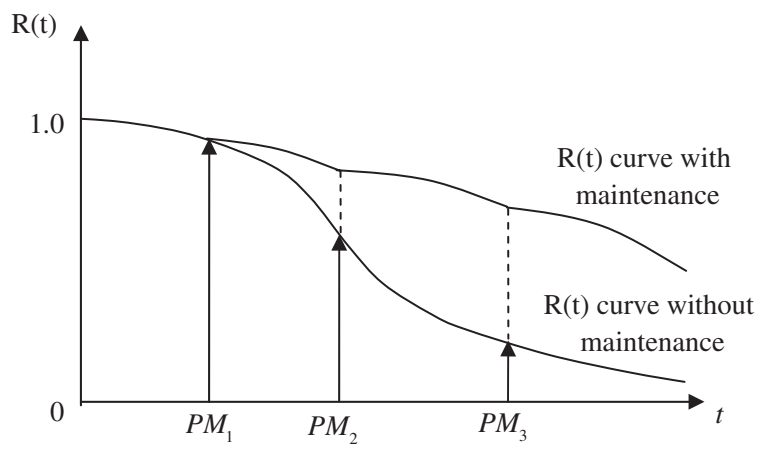

Figure 1. Reliability curve under minimal maintenance

Table I. Sampled data on the plant floor

\begin{tabular}{|c|c|c|c|c|c|c|}
\hline Machine & Set time & Clear time & Duration & $\begin{array}{c}\text { State } \\
\text { description }\end{array}$ & $\begin{array}{c}\text { Traditional } \\
\text { method }\end{array}$ & $\begin{array}{c}\text { Proposed } \\
\text { method }\end{array}$ \\
\hline M2 & $22: 30: 00$ & $22: 33: 43$ & $0: 03: 43$ & Faulted & $\mathrm{F}$ & $\mathrm{F}$ \\
\hline M2 & $22: 33: 43$ & $22: 35: 53$ & $0: 02: 10$ & In cycle & & \\
\hline M2 & $22: 35: 53$ & $22: 38: 10$ & $0: 02: 17$ & Faulted & $\mathrm{F}$ & $\mathrm{F}$ \\
\hline M2 & $22: 38: 10$ & $22: 53: 06$ & $0: 14: 56$ & In cycle & & \\
\hline M2 & $22: 53: 06$ & 23:03:11 & 0:10:05 & Pm (Oil change) & $\mathrm{R}$ & M \\
\hline M2 & 23:03:11 & $23: 15: 34$ & $0: 12: 23$ & In cycle & & \\
\hline M2 & $23: 15: 34$ & $23: 21: 39$ & 0:06:05 & Faulted & $\mathrm{F}$ & $\mathrm{F}$ \\
\hline M2 & $23: 21: 39$ & $23: 37: 23$ & $0: 15: 44$ & In cycle & & \\
\hline M2 & $23: 37: 23$ & $00: 21: 24$ & $0: 44: 01$ & Replacement & $\mathrm{R}$ & $\mathrm{R}$ \\
\hline M2 & $00: 21: 24$ & $00: 27: 49$ & $0: 06: 25$ & In cycle & & \\
\hline M2 & $00: 27: 49$ & $00: 30: 46$ & $0: 02: 57$ & Faulted & $\mathrm{F}$ & $\mathrm{F}$ \\
\hline M2 & $00: 30: 46$ & $00: 32: 12$ & $0: 01: 26$ & In cycle & & \\
\hline
\end{tabular}

F represents failure, $\mathrm{R}$ represents right censoring, and $\mathrm{M}$ represents minimal maintenance.

restores to one after the replacement. On the other hand, the $\mathrm{mm}$ restores the equipment to the previous deterioration stage rather than perfect reliability. This is the reason why $\mathrm{mm}$ is particularly useful to improve equipment reliability when the reliability distribution has an increasing hazard rate. In a normal situation, the difference and the effect when mm tasks are performed can be illustrated in Figure 1. The proposed reliability estimation method will consider the actual performance of $\mathrm{mm}$ tasks to obtain more accurate estimation results.

A data set segment sampled on the plant floor as shown in Table I can be used to further illustrate the difference in dealing with the data between the traditional methods and proposed reliability estimation methods. It can be seen that the proposed method distinguishes $\mathrm{mm}$ from $\mathrm{Mm}$, whereas the traditional method treats them as the same.

In order to calculate the maximum likelihood estimators based on the three types of data: failure, $\mathrm{Mm}$ and $\mathrm{mm}$, the historical data log can be divided into a number of segments. The boundaries of segments are successive time points of $\mathrm{RM}$ and $\mathrm{Mm}$, i.e. times when the reliability is restored to one. In the illustration in Figure 2, the whole data set is divided into four segments by failure or right censoring, and multiple mm tasks may occur in each segment.

\subsection{General reliability estimation procedures for operational data}

Based on the characteristics of operational data, the general procedures for proposed reliability estimation method are summarized as follows:

Step 1: Rough non-parametric estimation based on raw data to select parametric distributions. 


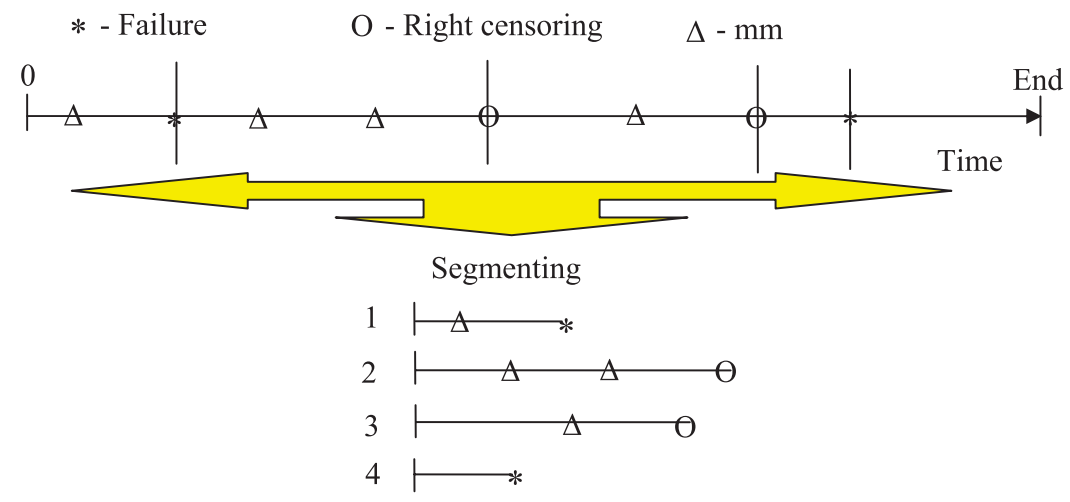

Figure 2. Example for raw data segmenting

Step 2: Rough parametric estimation among selected parametric distributions to decide whether a sample of data comes from a population with a specific distribution.

Step 3: Efficient parametric estimation for the specific distribution based on the MLE method in the presence of maintenance events.

For Step 1, the 'non-parametric' estimation is a simple statistical method based on the binomial distribution to estimate a cumulative distribution function $c d f F(t)$ from the censored data ${ }^{1}$. This method is generally applicable but is not very accurate.

We use rough estimates because several assumptions are routinely made in traditional estimation methods when performing the non-parametric or parametric estimation:

1. All PM tasks including $\mathrm{mm}$ and $\mathrm{Mm}$ are considered as right censoring, and the reliability of the system is recovered to one after the task is completed.

2. After the system fails, the fixed machine is in perfect condition and the reliability is also back to one.

3. The sampled data are independent of each other.

Obviously, the mm information is not well considered in such an approach and hence the non-parametric estimation can be used only for rough estimation based on simplified operational data. With the rough non-parametric estimation, the trends of hazard rate and $c d f$ are found, which may be used to select the right parametric models. The Kaplan-Meier non-parametric estimator is frequently used for the calculation.

For Step 2, as the non-parametric estimation is usually used for subsequent identification of appropriate parametric models, one needs to select a parametric distribution for further analysis. Weibull distribution is often used to describe the equipment reliability ${ }^{1}$. The Anderson-Darling (AD) test ${ }^{16}$ can be used to test whether a sample of data is part of a population with a specific distribution. The smaller the AD value, the better the fit. Following the same assumptions as those in non-parametric estimation, the rough parametric estimation can be performed.

To increase the accuracy of estimation instead of 'rough' results, further calculations in Step 3 based on the MLE are required. The general procedure for performing this efficient parametric estimation has been obtained based on the reliability curve in the presence of maintenance events especially $\mathrm{mm}$ tasks as in Figure 3.

First, let us assume that for the segment $i$ between $t_{i, 0}$ and $t_{i, 1}$ with mm tasks 1 to $N_{i}$ shown in Figure 3 , we have the reliability function $R_{P M}\left(t_{i, 1}\right)$ and probability density function $f_{P M}\left(t_{i, 1}\right)$.

Second, based on these results, the likelihood function in segment $\left(t_{i, 0}, t_{i, 1}\right)$ can be expressed as

$$
\begin{aligned}
L_{i}\left(\theta_{1}, \theta_{2}, \ldots, \theta_{P}, \text { data }_{i}\right) & =f_{P M}\left(t_{i, 1}\right)^{\delta_{i}} R_{P M}\left(t_{i, 1}\right)^{\left(1-\delta_{i}\right)} \\
\delta_{i} & = \begin{cases}1 & \text { Exact failure at } t_{i, 1} \\
0 & \text { Right censoring at } t_{i, 1}\end{cases}
\end{aligned}
$$

where $\theta_{1}, \theta_{2}, \ldots, \theta_{P}$ are the $P$ parameters for a certain distribution. 


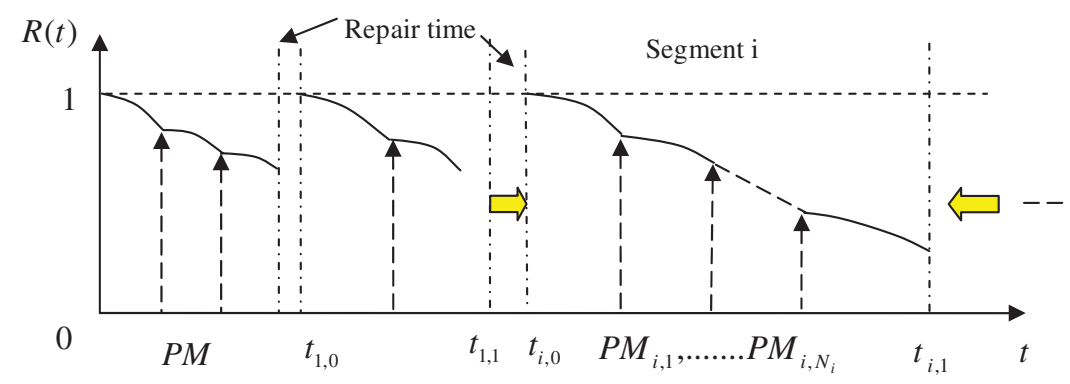

Figure 3. Reliability performance with operational data

Third, under the assumptions of independence of censoring and failures, the likelihood function for all segments becomes

$$
\begin{aligned}
L\left(\theta_{1}, \theta_{2}, \ldots, \theta_{P}\right) & =\prod_{i=1}^{n} L_{i}\left(\theta_{1}, \theta_{2}, \ldots, \theta_{P}, \text { data }_{i}\right)=\prod_{i=1}^{n} f_{P M}^{\delta_{i}} R_{P M}^{\left(1-\delta_{i}\right)} \\
\delta_{i} & = \begin{cases}1 & \text { Exact failure at } t_{i, 1} \\
0 & \text { Right censoring at } t_{i, 1}\end{cases}
\end{aligned}
$$

Last, by definition, the MLE for $\hat{\theta}_{1}$ to $\hat{\theta}_{P}$ can be calculated by solving the following system of equations:

$$
\begin{aligned}
& \frac{\partial \log \left[L\left(\theta_{1}, \theta_{2}, \ldots, \theta_{P}\right)\right]}{\partial \theta_{1}}=0 \\
& \frac{\partial \log \left[L\left(\theta_{1}, \theta_{2}, \ldots, \theta_{P}\right)\right]}{\partial \theta_{2}}=0 \\
& \frac{\partial \log \left[L\left(\theta_{1}, \theta_{2}, \ldots, \theta_{P}\right)\right]}{\partial \theta_{P}}=0
\end{aligned}
$$

for parameters $\hat{\theta_{1}}, \hat{\theta_{2}}, \ldots, \hat{\theta_{P}}$.

More details of the calculations for the commonly used Weibull distribution are present in Section 2.3 to demonstrate this procedure for efficient parametric estimation.

\subsection{Estimation for the case of Weibull distribution}

A two-parameter Weibull distribution can be characterized as follows:

The probability density function $p d f: f(t)=\frac{\beta}{\eta^{\beta}} t^{\beta-1} \mathrm{e}^{-(t / \eta)^{\beta}}, \quad t>0, \quad \eta>0, \quad \beta>0$.

The reliability function $R(t)=\mathrm{e}^{-(t / \eta)^{\beta}}$.

Parameter $\beta>0$ is referred to as the shape parameter, and $\eta>0$ is referred to as the scale parameter. When $\beta>1$, the corresponding Weibull distribution will have an increasing hazard rate. Such distribution depicts the reliability of actual equipment more realistically than the exponential distribution (constant hazard rate), because aging usually causes the equipment to deteriorate over time and thus have an increasing hazard rate.

For PM tasks, 1 to $N_{i}$ in period $t_{i, 0}$ to $t_{i, 1}$, the reliability $R_{P M}(t)$ and $p d f f_{P M}(t)$ over that period of time can be expressed as

$$
\begin{aligned}
& R_{P M}(t)=\mathrm{e}^{-\left(\frac{t_{i, P M, 1},-t_{i, 0}}{\eta}\right)^{\beta}} \mathrm{e}^{-\left(\frac{t_{i, P M, 2}-t_{i, P M, 1}}{\eta}\right)^{\beta}} \cdots \mathrm{e}^{-\left(\frac{t-t_{i, P M, m}}{\eta}\right)^{\beta}} \\
& f_{P M}(t)=\mathrm{e}^{-\left(\frac{t_{i, P M, 1}-t_{i, 0}}{\eta}\right)^{\beta}} \mathrm{e}^{-\left(\frac{t_{i, P M, 2}-t_{i, P M, 1}}{\eta}\right)^{\beta}} \cdots \mathrm{e}^{-\left(\frac{t-t_{i, P M, m}}{\eta}\right)^{\beta}} \frac{\beta}{\eta^{\beta}}\left(t-t_{i, P M, m}\right)^{\beta-1}
\end{aligned}
$$

where $t_{i, P M, m}, m \in\left[1, N_{i}\right]$, is the time when the $m$ th PM task is performed. 
The reliability $R_{P M}\left(t_{i, 1}\right)$ and $p d f f_{P M}\left(t_{i, 1}\right)$ can be expressed as

$$
\begin{aligned}
& R_{P M}\left(t_{i, 1}\right)=\mathrm{e}^{-\left(\frac{t_{i, P M, 1},-t_{i, 0}}{\eta}\right)^{\beta}} \mathrm{e}^{-\left(\frac{t_{i, P M, 2}-t_{i, P M, 1}}{\eta}\right)^{\beta}} \cdots \mathrm{e}^{-\left(\frac{t_{i, 1}-t_{i, P M, N_{i}}}{\eta}\right)^{\beta}} \\
& f_{P M}\left(t_{i, 1}\right)=\mathrm{e}^{-\left(\frac{t_{i, P M, 1},-t_{i, 0}}{\eta}\right)^{\beta}} \mathrm{e}^{-\left(\frac{t_{i, P M, 2}-t_{i, P M, 1}}{\eta}\right)^{\beta}} \cdots \mathrm{e}^{-\left(\frac{t_{i, 1}-t_{i, P M, N i}}{\eta}\right)^{\beta}} \frac{\beta}{\eta^{\beta}}\left(t_{i, 1}-t_{i, P M, N_{i}}\right)^{\beta-1}
\end{aligned}
$$

Based on these results, the likelihood function in segment $\left(t_{i, 0}, t_{i, 1}\right)$ is obtained as

$$
\begin{aligned}
L_{i}\left(\beta, \eta, \text { data }_{i}\right)= & f_{P M}\left(t_{i, 1}\right)^{\delta_{i}} R_{P M}\left(t_{i, 1}\right)^{\left(1-\delta_{i}\right)} \\
= & {\left[\mathrm{e}^{-\left(\frac{t_{i, P M, 1},-t_{i, 0}}{\eta}\right)^{\beta}} \mathrm{e}^{-\left(\frac{t_{i, P M, 2}-t_{i, P M, 1}}{\eta}\right)^{\beta}} \cdots \mathrm{e}^{-\left(\frac{t_{i, 1}-t_{i, P M, N_{i}}}{\eta}\right)^{\beta}} \frac{\beta}{\eta^{\beta}}\left(t_{i, 1}-t_{i, P M, N_{i}}\right)^{\beta-1}\right]^{\delta_{i}} } \\
& \times\left[\mathrm{e}^{-\left(\frac{t_{i, P M, 1},-t_{i, 0}}{\eta}\right)^{\beta}} \mathrm{e}^{-\left(\frac{t_{i, P M, 2}-t_{i, P M, 1}}{\eta}\right)^{\beta}} \cdots \mathrm{e}^{-\left(\frac{t_{i, 1}-t_{i, P M, N i}}{\eta}\right)^{\beta}}\right]^{\left(1-\delta_{i}\right)} \\
\delta_{i}= & \begin{cases}1 & \text { Exact failure at } t_{i, 1} \\
0 & \text { Right censoring at } t_{i, 1}\end{cases}
\end{aligned}
$$

Therefore, the likelihood function for all segments putting together becomes

$$
\begin{aligned}
& L(\beta, \eta)=\prod_{i=1}^{n} L_{i}\left(\beta, \eta, \text { data }_{i}\right)=\prod_{i=1}^{n} f_{P M}^{\delta_{i}} R_{P M}^{\left(1-\delta_{i}\right)} \\
& =\prod_{i=1}^{n}\left[\mathrm{e}^{-\left(\frac{t_{i, P M, 1},-t_{i, 0}}{\eta}\right)^{\beta}} \mathrm{e}^{-\left(\frac{t_{i, P M, 2}-t_{i, P M, 1}}{\eta}\right)^{\beta}} \cdots \mathrm{e}^{-\left(\frac{t_{i, 1}-t_{i, P M, N_{i}}}{\eta}\right)^{\beta}} \frac{\beta}{\eta^{\beta}}\left(t_{i, 1}-t_{i, P M, N_{i}}\right)^{\beta-1}\right]^{\delta_{i}} \\
& \times\left[\mathrm{e}^{-\left(\frac{t_{i, P M, 1},-t_{i, 0}}{\eta}\right)^{\beta}} \mathrm{e}^{-\left(\frac{t_{i, P M, 2}-t_{i, P M, 1}}{\eta}\right)^{\beta}} \cdots \mathrm{e}^{-\left(\frac{t_{i, 1}-t_{i, P M, N_{i}}}{\eta}\right)^{\beta}}\right]^{\left(1-\delta_{i}\right)} \\
& \delta_{i}= \begin{cases}1 & \text { Exact failure at } t_{i, 1} \\
0 & \text { Right censoring at } t_{i, 1}\end{cases}
\end{aligned}
$$

Subsequently, the MLE for $\eta$ and $\beta$ can be obtained by solving the following equations for parameters $\hat{\beta}$ and $\hat{\eta}$ :

$$
\begin{aligned}
& \frac{\partial \log [L(\beta, \eta)]}{\partial \beta}=0 \\
& \frac{\partial \log [L(\beta, \eta)]}{\partial \eta}=0
\end{aligned}
$$

Parameter estimates $\hat{\beta}$ and $\hat{\eta}$ can be evaluated by nonlinear optimization method, which will be further discussed in the case study.

\section{NUMERICAL EXAMPLES}

Numerical examples are performed to illustrate that the proposed reliability estimation method could find a given actual distribution. The process for this application is illustrated in Figure 4. Monte Carlo simulation 


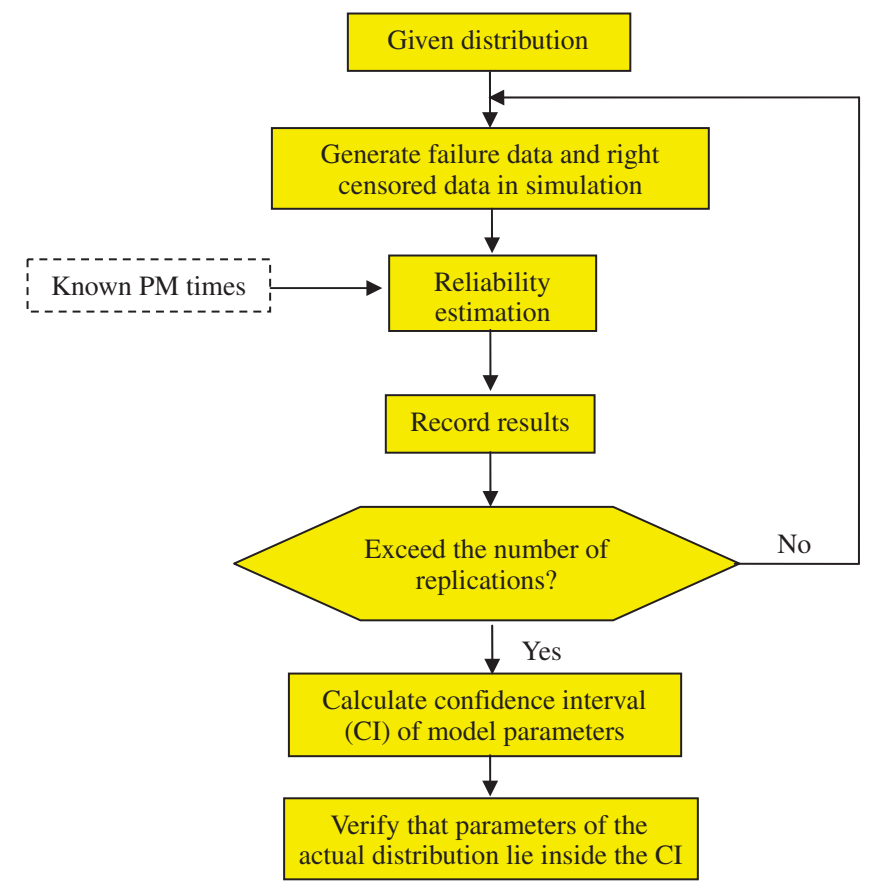

Figure 4. Flow chart to apply the proposed method in simulated data

is used to generate the failure-time data and censored data for a given distribution with known PM time. The Monte Carlo simulation is a method for iteratively evaluating a deterministic model using sets of random numbers as inputs. This method is often used when the model is complex, nonlinear, or involves more than just a couple of uncertain parameters. A simulation can typically involve over 10000 evaluations of the model, a task which in the past was only practical using super computers. The reliability parameters can then be estimated by the proposed estimation procedures as mentioned in Section 2.2. The confidence interval (CI) for the estimated parameter is obtained by many replications. Finally, we compare the performances to validate the given parameters to lie within the obtained CI.

Table II summarizes the results of 30 such simulated replications. It is known that the actual equipment reliability follows a Weibull distribution with parameters $\eta=5672.4$ and $\beta=4.38$ (these parameters are obtained from the reliability analysis on failing SH-60 helicopter gearbox). In this case, 1000 data including failures and right censoring are generated randomly for each replication.

The mean value and the standard deviation of the 30 replications can be calculated as $\bar{\eta}=5733.38, \bar{\beta}=4.45$ and $S_{\eta}=336.78, S_{\beta}=0.43$. The $95 \%$ CI can be calculated using the equation $\bar{x} \pm z_{\alpha / 2} * s / \sqrt{n}$

$$
C I_{\eta, 0.95}=[5612.86,5853.88], \quad C I_{\beta, 0.95}=[4.27,4.64]
$$

It is obvious that the actual parameters $\eta=5672.4 \in C I_{\eta, 0.95}$, and $\beta=4.38 \in C I_{\beta, 0.95}$, which demonstrates that the proposed method can find a given distribution. Similarly, hundreds of numerical case studies have been performed to validate the proposed reliability estimation method.

\section{INDUSTRIAL CASE STUDY}

The newly proposed method of reliability estimation has been implemented on an automotive assembly line of a local automotive corporation. The layout of the assembly line along with cycle time and buffer capacity is shown in Figure 5. There are five types of raw work pieces (kick up, real floor, center floor, front 
Table II. Results of reliability estimation in 30 replications

\begin{tabular}{llcccc}
\hline Replication & $\hat{\eta}$ & $\hat{\beta}$ & Replication & $\hat{\eta}$ & $\hat{\beta}$ \\
\hline 1 & 6258.6 & 4.58 & 16 & 5554.9 & 4.11 \\
2 & 5551.8 & 5.01 & 17 & 5845.4 & 3.76 \\
3 & 5994.4 & 4.32 & 18 & 5146.1 & 4.47 \\
4 & 5705.9 & 4.64 & 19 & 6119.8 & 4.57 \\
5 & 5315.1 & 4.21 & 20 & 5647.5 & 4.18 \\
6 & 5700.3 & 4.56 & 21 & 5705.2 & 4.03 \\
7 & 5680.2 & 4.72 & 22 & 6303.6 & 5.18 \\
8 & 6323.1 & 4.81 & 23 & 5408.1 & 4.19 \\
9 & 5315.7 & 4.71 & 24 & 5851.2 & 4.66 \\
10 & 6037.4 & 4.52 & 25 & 5826.8 & 5.06 \\
11 & 6217 & 3.69 & 26 & 5392.4 & 4.54 \\
12 & 5906.1 & 4.71 & 27 & 5663.7 & 3.75 \\
13 & 5520.4 & 4.18 & 28 & 5835.1 & 4.94 \\
14 & 5142.9 & 4.08 & 29 & 5219.9 & 3.97 \\
15 & 5955 & 5.36 & 30 & 5857.4 & 4.08 \\
\hline
\end{tabular}

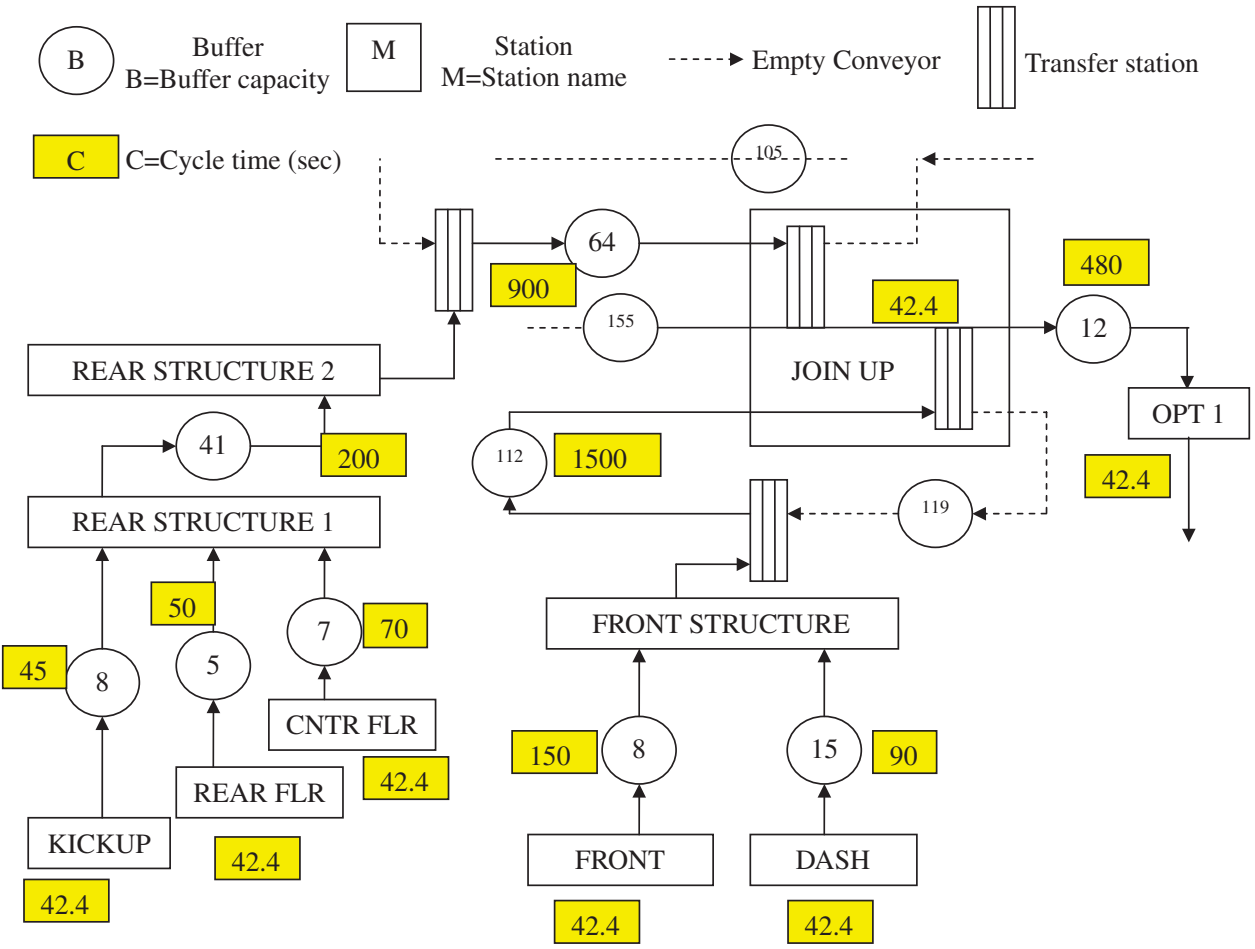

Figure 5. Layout and parameters of a production line

and dash) inputted from stations M1-M5, respectively, 10 stations (M1-M10), and 12 buffer locations. The assembly line is used for the production of the doors of the car. The following production parameters were obtained from the manufacturers:

- cycle time of each station;

- moving speed and logic of each conveyor/bank;

- conveyor/bank count log of one shift;

- production downtime log and PM schedule. 

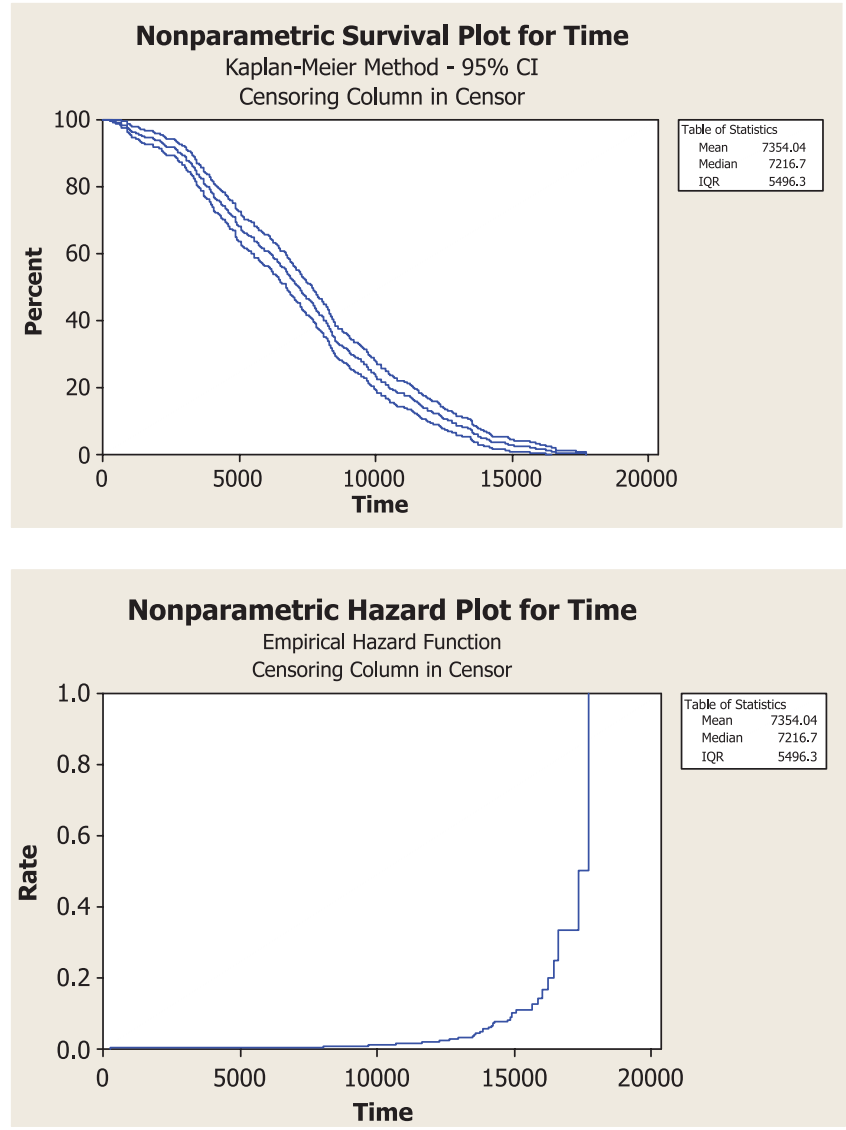

Figure 6. Rough non-parametric estimation results

As the data set segment illustrated in Table I, a two-month operational data log obtained from workstation M2 is used to implement the proposed reliability estimation method. The data were sampled by the company internal information systems in the plant: factory information system and basic communication system. The procedures of implementation are summarized as follows:

Step 1: The results of applying the Kaplan-Meier non-parametric estimator on the data set are shown in Figure 6. These rough non-parametric estimation results are obtained under the assumptions stated in Section 2.2. The results show an increasing hazard rate with a smooth change. Therefore, a single distribution such as a Weibull distribution or a lognormal distribution can be used. The exponential distribution is not appropriate in this case because the data obviously do not display a constant hazard rate.

Step 2: Rough parametric estimation is used for selecting the right parametric distribution using the MLE method with the same assumptions as in Step 1. On performing the rough parametric estimations on the commonly used Weibull distribution and lognormal distribution, the results shown in Figure 7 are obtained.

The $\mathrm{AD}$ values for these distributions are calculated as $A D_{\text {Weibull }}=0.0614<A D_{\text {Lognormal }}=8.122$. As the smallest $\mathrm{AD}$ value corresponds to the Weibull distribution, a conclusion can be drawn that Weibull distribution is a better fit for this data set. Therefore, Weibull distribution is selected for the calculation of accurate estimated parameters.

Step 3: Efficient parametric estimation is performed on the Weibull distribution based on the MLE method mentioned in Section 2.3. Through constrained nonlinear optimization or nonlinear programming ${ }^{17,18}$ implemented in Matlab (http://www.mathworks.com), equations in Section 2.3 can be solved as $\hat{\eta}=8674.4$ and $\hat{\beta}=1.85$ in this case. 

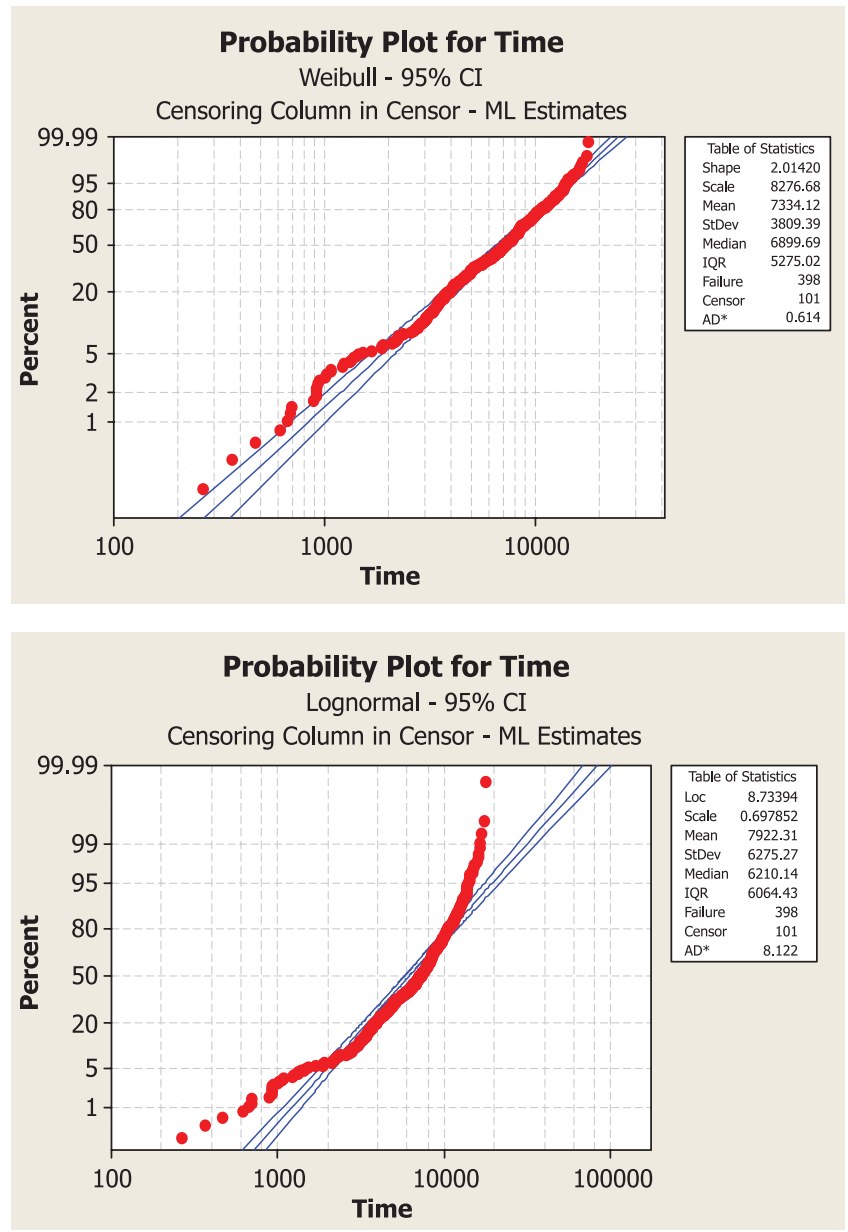

Figure 7. Rough parametric estimation results

To compare these results with those of traditional estimation methods obtained in the first picture of Figure $7\left(\hat{\eta}_{\text {Traditional }}=8276.68\right.$ and $\hat{\beta}_{\text {Traditional }}=2.0142$ for Weibull distribution $)$, the mean time to failure (MTTF) is calculated for Weibull distribution by

$$
M T T F=\eta \cdot \Gamma(1+1 / \beta)
$$

where $\Gamma$ represents gamma distribution.

Therefore, $M T T F_{N e w \_m e t h o d}=7686.543 \mathrm{~s}$ for the proposed method with $\hat{\eta}=8674.4$ and $\hat{\beta}=1.85$, and $M T T F_{\text {Traditional_method }}=8592.927 \mathrm{~s}$ for the traditional method with $\hat{\eta}_{\text {Traditional }}=8276.68$ and $\hat{\beta}_{\text {Traditional }}=$ 2.0142. These calculation results show that the traditional reliability estimation method on the plant floor overestimates the equipment reliability, which may cause safety problem and severely damage the equipment.

The reliability estimation results are further utilized and applied for maintenance decision making on the plant floor: if the reliability of a machine is detected lower than the threshold $R$, the PM work-order is triggered to improve the equipment reliability. The threshold for failure alarm may be set to well-accepted standards such as International Organization for Standardization (ISO), the British diagnostics company VCI ltd, or the American IRD. In this manner, the catastrophic failure could be prevented. The results in Table III show that this reliability-based maintenance policy improves system reliability and leads to $4.44 \%$ throughput increment in 1 week ( 5 working days) compared with base line performance in the plant. For 
Table III. Comparison of the proposed maintenance method with baseline

\begin{tabular}{lcc}
\hline Maintenance policy & Average throughput (parts/week) & Throughput increment (\%) \\
\hline Base line & 6921 & - \\
Reliability-based policy & 7243 & 4.45 \\
\hline
\end{tabular}

baseline, only RM tasks in first come first serve are considered wherein maintenance engineers perform maintenance only on failed machines in the order in which they fail.

In traditional practice on the plant floor, exponential distribution is widely used because of its simple format. The main parameters describing equipment failures, mean time to repair and mean time between failures, are assumed to follow exponential distribution. However, exponential distribution has a constant hazard rate, which is not practical because aging and wear will make the equipment have an increasing hazard rate in reality. Therefore, Weibull distribution is more suited to describe the reliability performance than the exponential distribution. The accuracy of reliability estimation greatly depends on the sampled data on the plant floor. Although most current data acquisition systems on the plant floor have the ability to collect the needed data, many junk or noise data are still included. Data filtering and cleaning is necessary before performing parameter estimation. Furthermore, sensor degradation problem is usually ignored in the plant, and only failed sensors will be replaced. Unfortunately, data from degraded sensor could not accurately describe the current system performance, which causes inaccurate reliability estimation.

\section{CONCLUSIONS AND FUTURE WORKS}

Many traditional maintenance models and reliability analysis methods usually assume that only perfect maintenance is performed on the system and the system will restore to as good as new regardless of the kind of PM work-order that is performed. This is not practical in reality. In this paper, maximum likelihood parameter estimation in the presence of failure time and maintenance logs corresponding to an equipment is studied to obtain more accurate and safer reliability estimations than the traditional methods. The difference in reliability effect between $\mathrm{Mm}$ and $\mathrm{mm}$ is considered. The general procedure for reliability estimation based on operational data is presented, and detailed equations for Weibull distribution are obtained. Both numerical case study and real industrial application are performed to validate the proposed reliability estimation method. Numerical case study illustrates that a given distribution can be found by the proposed method. The industrial implementation has also been performed on a production line in an automotive assembly plant. The results show that the traditional reliability estimation methods produce an overestimate of the system reliability, and the newly proposed method can improve the production safety and reliability. The achieved reliability model is utilized for further work on the plant floor such as estimating the remaining useful life of equipment or scheduling and prioritizing maintenance work-orders.

Although the single Weibull distribution works well in most cases, mixed Weibull distribution is possibly an approximate distribution to use in modelling the lifetimes of the units that have more than one failure mode. The method can later be expanded to mixed distributions.

\section{REFERENCES}

1. Meeker WQ, Escobar LA. Statistical Methods for Reliability Data. Wiley: New York, 1998.

2. Lisnianski A, Levitin G, Ben-Haim H. Structure optimization of multi-state system with time redundancy. Reliability Engineering and System Safety 2000; 67:103-112.

3. Wu SM, Clements-Croome D. Optimal maintenance policies under different operational schedules. IEEE Transactions on Reliability 2005; 54(2):338-346. 
4. Hossain AM, Zimmer WJ. Comparison of estimation methods for Weibull parameters: Complete and censored samples. Journal of Statistical Computation and Simulation 2003; 73(2):145-153.

5. Cox DR, Hinkley DV. Theoretical Statistics. Chapman \& Hall: London, 1974.

6. Lindsey JK. Parametric Statistical Inference. Oxford University Press: Oxford, 1990.

7. Zhang LF, Xie M, Tang LC. Robust regression using probability plots for estimating the Weibull shape parameter. Quality and Reliability Engineering International 2006; 22:905-917.

8. Montanari GC, Mazzanti G, Cacciari M, Fothergill JC. In search of convenient techniques for reducing bias in the estimation of Weibull parameter for uncensored tests. IEEE Transactions on Dielectrics and Electrical Insulation 1997; 4(3):306-313.

9. Ross R. Formulas to describe the bias and standard deviation of the ML estimated Weibull shape parameter. IEEE Transactions on Dielectrics and Electrical Insulation 1994; 1(2):247-253.

10. Ross R. Bias and standard deviation due to Weibull parameter estimation for small data sets. IEEE Transactions on Dielectrics and Electrical Insulation 1996; 3(1):28-42.

11. Hirose H. Bias correction for the maximum likelihood estimates in the two parameter Weibull distribution. IEEE Transactions on Dielectrics and Electrical Insulation 1999; 6(1):66-68.

12. Zhang LF, Xie M, Tang LC. Bias correction for the least squares estimator of Weibull shape parameter with complete and censored data. Reliability Engineering and System Safety 2006; 91:930-939.

13. Zhou X, Xi L, Lee J. Reliability-centered predictive maintenance scheduling for a continuously monitored system subject to degradation. Reliability Engineering and System Safety 2007; 92:530-534.

14. Sim SH, Endrenyi J. A failure-repair model with minimal \& major maintenance. IEEE Transactions on Reliability 1993; 42(1):134-140.

15. Hosseini MM, Kerr RM, Randall RB. An inspection model with minimal and major maintenance for a system with deterioration and Poisson failures. IEEE Transactions on Reliability 2000; 49(1):88-98.

16. Stephens MA. EDF statistics for goodness of fit and some comparisons. Journal of the American Statistical Association 1974; 69:730-737.

17. Coleman TF, Li Y. An interior, trust region approach for nonlinear minimization subject to bounds. SIAM Journal on Optimization 1996; 6:418-445.

18. Coleman TF, Li Y. On the convergence of reflective Newton methods for large-scale nonlinear minimization subject to bounds. Mathematical Programming 1994; 67(2):189-224.

\section{Authors' biographies}

Lin Li received his $\mathrm{PhD}$ in Mechanical Engineering from the University of Michigan-Ann Arbor in 2007. Currently, he is a research fellow in the S. M. Wu Manufacturing Research Center at the University of Michigan. His research interests include design, control and simulation of manufacturing systems, production research, intelligent maintenance, reliability engineering, and cost model for joint production and maintenance.

Jun Ni received his PhD in Mechanical Engineering from the University of Wisconsin-Madison in 1987. Currently, he is a Professor in the Department of Mechanical Engineering at the University of MichiganAnn Arbor. He is the Director of S. M. Wu Manufacturing Research Center, the Co-Director of MultiCampus National Science Foundation Center for Intelligent Maintenance Systems, and Shien-Ming (Sam) Wu Collegiate Professor of Manufacturing. His research interest includes Manufacturing process modeling, analysis and prediction; precision engineering and metrology; cutting tool development, quality control methods, intelligent maintenance systems, monitoring and fault diagnosis. He has over 200 publications in refereed journals and conferences. He is an elected fellow of ASME. 